\title{
Long-period Red Variables in the Large Magellanic Cloud from the MOA Database
}

\author{
Mine Takeuti \\ Astron. Inst., Tohoku Univ., Sendai, 980-8578 Japan
}

Noda, S.

Solar-Terrestrial Environment Lab., Nagoya Univ., Nagoya, 464-8601 Japan

Abe, F. ${ }^{1}$ Bond, I. A. ${ }^{2}$, Dodd, R. J. ${ }^{3}$, Hearnshaw, J. B. ${ }^{4}$, Honda, M. ${ }^{5}$, Jugaku, J. ${ }^{6}$, Kabe, S. ${ }^{7}$, Kilmartin, P. M. ${ }^{2,4}$, Matsubara, Y. ${ }^{1}$, Masuda, K. ${ }^{1}$, Muraki, Y. ${ }^{1}$, Nakamura, T. ${ }^{8}$, Nankivell, G. R. ${ }^{9}$, Noguchi, C. ${ }^{1}$, Ohnishi, K. ${ }^{10}$, Rattenbury, N. J. ${ }^{2}$, Reid, M. ${ }^{11}$, Saito, To. ${ }^{12}$, Sato, H. ${ }^{8}$, Sekiguchi, M. ${ }^{7}$, Skuljan, J. ${ }^{4}$, Sullivan, D. J. ${ }^{11}$, Sumi, T. ${ }^{1}$, Watase, Y. ${ }^{7}$, Yanagisawa, T. ${ }^{1}$, Yock, P. C. M. ${ }^{2}$, and Yoshizawa, M. ${ }^{13}$

Abstract. We studied 147 long-period red variable stars in the Large Magellanic Cloud from the MOA database. Amongst them, seven red luminous stars are likely pulsating in a higher mode.

\section{Observation and Reduction}

The MOA is a massive photometry project designed to study the distribution of cosmic dark matter, based on observations of gravitational microlensing events.

\footnotetext{
${ }^{1}$ Solar-Terrestrial Environment Lab., Nagoya Univ., Nagoya, 464-8601 Japan

${ }^{2}$ Dept. of Physics, Univ. of Auckland, Auckland, New Zealand

${ }^{3}$ Carter National Obs., Wellington, New Zealand

${ }^{4}$ Dept. of Physics and Astron., Univ. of Canterbury, Christchurch, New Zealand

${ }^{5}$ Inst. Cosmic Ray Research, Univ. of Tokyo, Kashiwa, 277-8582 Japan

${ }^{6}$ Institute for Civilization, Tokai Univ., Tokyo, Japan

${ }^{7}$ KEK, Tsukuba, 305-0801 Japan

${ }^{8}$ Research Inst. Fundamental Physics, Kyoto Univ., Kyoto, 606-8502 Japan

${ }^{9}$ Lower Hutt, New Zealand

${ }^{10}$ Nagano Tech. College, Nagano, Japan

${ }^{11}$ Dept. of Physics, Victoria Univ., Wellington, New Zealand

${ }^{12}$ Tokyo Metropolitan College of Aeronautics, Tokyo, 140-0011 Japan

${ }^{13}$ National Astron. Obs., Mitaka, Tokyo, 181-8588 Japan
} 
Millions of stars in the Large Magellanic Could are monitored by using a widefield camera on a 0.61-m telescope at the Mount John Observatory of Canterbury University on the South Island of New Zealand.

Variable star research with the MOA project has been described in Hearnshaw et al. (2000). In a recent study, we compare our results with those of the WFPC2 of the HST taking the effects of colour into account. Calibration is performed by a careful frame-to-frame check (Kato 2000). Approximately 300 observations, from January 1997 to December 1999, are used. The probable error of the intensity mean, $\left\langle V_{m}\right\rangle$ and $\left\langle R_{m}\right\rangle$ is less than \pm 0.015 mag. We have analysed the $V_{m}$ and $R_{m}$ periodicity of stars that show a prominent variability by using a folding method, the phase difference minimization. Variables with a period of less than $30 \mathrm{~d}$ or with colour $\left\langle V_{m}\right\rangle-\left\langle R_{m}\right\rangle$ less than 0.4 are omitted. Because our time span is $1060 \mathrm{~d}$, we also omitted stars with periods longer than $400 \mathrm{~d}$. Finally, 147 stars with an amplitude $\Delta R$ larger than 1.3 mag are selected. Because the blue passband of the MOA system is very broad, the relation among the $V_{m}, R_{m}$, and $K$-magnitudes is different for M-type and C-type stars, due to the effect of the TiO band.

\section{Results}

The scattering of the period-colour diagram is smaller than in our preliminary report (Takeuti et al. 2000). Among the 147 stars, seven red and short-period variables are separated from the other variables. The $K$-magnitude- $\log P$ relation of M-Miras coincides with that presented by Feast et al. (1989). On this diagram, the seven stars form a group separated from the other stars. On the $K$ magnitude-colour diagram, the stars locate at the tip of the giant branch. This indicates that the radii of these stars are very large. These large, short-period stars are less regular in their periodicity, and their amplitudes are small. They may be a counterpart of the short period red (SP red) stars found in the study of Hipparcos parallaxes (Whitelock \& Feast 2000), and also the stars suggested to be higher mode pulsators (Wood \& Sebo 1996; Bedding \& Zijlstra 1998).

\section{References}

Bedding, T. R., \& Zijlstra, A. A. 1998, ApJ, 506, L47

Feast, M. W., Glass, I. S., Whitelock, P. A., \& Catchpole, R. M. 1989, MNRAS, 241,375

Hearnshaw, J. B., Bond, I. A., Rattenbury, N. J., et al. 2000, in ASP Conf. Ser. Vol. 203, The Impact of Large-scale Surveys on Pulsating Star Research, ed. L. Szabados, D. W. Kurtz (San Francisco: ASP), 203, 31

Kato, Y. 2000, Dissertation for Master Degree, Nagoya University (in Japanese)

Takeuti, M., Noda, S., Bond, I. A., et al. 2000, in ASP Conf. Ser. Vol. 203, The Impact of Large-scale Surveys on Pulsating Star Research, ed. L. Szabados, D. W. Kurtz (San Francisco: ASP), 120

Whitelock, P., \& Feast, M. 2000, MNRAS, 319, 759

Wood, P. R., \& Sebo, K. M. 1996, MNRAS, 282, 958 